\title{
Existence of a global weak solution to a Compressible Primitive Equations.
}

\author{
Mehmet Ersoy $* \uparrow 1,2,3$ and Timack Ngom $\$ 4$ \\ ${ }^{2}$ Basque Center for Applied Mathematics (BCAM), Bizkaia Technology Park 500, , 48160, Derio, Basque Country, \\ Spain \\ ${ }^{3}$ Present address: Institut de Mathématique de Toulon (IMATH), Université du Sud Toulon-Var, Av. G. Pompidou \\ BP 56, , 83162 La Valette du Var, France \\ ${ }^{4}$ Laboratoire d'Analyse Numérique et Informatique(LANI), Université Gaston Berger de Saint-Louis, UFR SAT, BP \\ 234, , Saint-Louis,Sénégal \\ ${ }^{1}$ Laboratoire de Mathématiques (LAMA), Université de Savoie, , 73376 Le Bourget du Lac, France
}

18 avril 2012

\begin{abstract}
Résumé
Abstract

In this note, we show a global weak existence result for a two dimensional Compressible Primitive Equations for atmosphere dynamics modeling.

\section{Résumé}

Existence d'une solution faible pour un modèle d'Équations Primitives Compressibles

Dans cette Note, on montre un résultat d'existence de solutions faibles globale en temps pour un modèle d'Équations Primitives Compressibles en dimension deux pour la dynamique de l'atmosphère.
\end{abstract}

\section{Introduction}

The Primitive Equations (PEs) of the atmosphere modeling are fundamental equations of geophysical fluid mechanics (see, e.g., Pedlowski [6]). In the hierarchy of models for geophysical flow, the PEs are situated between the so-called non hydrostatic models and shallow water models. They are generally derived from the full set of geophysical fluid equations. Owing to the difference of the depth and length scale, the derivation consists in replacing the momentum conservation equation for the vertical velocity by the hydrostatic equation, in the same spirit of the derivation of the shallow water equations (see, for instance, Gerbeau and Perthame [3] or Marche [5]). We refer to the works by Lions, Temam and Wang [4] for the mathematical formulation and existence results for these equations.

In this paper, we investigate a simple version of the Compressible Primitive Equations (CPEs) for atmosphere dynamics where we do not deal with complexe phenomena as solar heating effects or the amount of water in the air (as done by Lions, Temam and Wang [4] or Temam and Ziane [7]). This model is already introduced by Gatapov and Kazhikhov [2], and they obtain a global existence theorem for weak solutions for a model, called model problem, close to the one presented in this note. As a straightforward consequence of

\footnotetext{
*The work is supported by the ERC Advanced Grant FP7-246775 NUMERIWAVES

$\dagger$ e-mail: Mehmet.Ersoy@univ-tln.fr

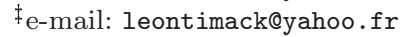


the existence result [2], we prove the global solvability of the initial boundary value problem for the simplified CPEs.

\section{A global existence result for the simplified CPEs}

We start with the simplified version of CPEs which, in cartesian coordinates, reads:

$$
\begin{cases}\partial_{t} \rho+\partial_{x}(\rho u)+\partial_{y}(\rho v) & =0 \\ \partial_{t}(\rho u)+\partial_{x}\left(\rho u^{2}\right)+\partial_{y}(\rho u v)+\partial_{x} p & =\partial_{x}\left(\nu_{1} \partial_{x} u\right)+\partial_{y}\left(\nu_{2} \partial_{y} u\right) \\ \partial_{y} p & =-\rho g\end{cases}
$$

where $x, y$ stand for the horizontal and the vertical space variables. $\rho$ is the density, $\mathbf{u}=(u, v)$ is the velocity of the fluid with $u$ (resp. $v$ ) the horizontal (resp. vertical) component, $p$ is the pressure given by the equation of state $p(\rho)=c^{2} \rho$ for some given constant $c$,

The constant $c$ is usually $c^{2}=\mathcal{R} \mathcal{T}$ where $\mathcal{R}$ is the specific gas constant for the air and $\mathcal{T}$ is the temperature, assumed constant. The turbulence viscosities are $\left(\nu_{1}, \nu_{2}\right)$ for the horizontal and vertical direction and is written: $\nu_{1}(t, x, y)=\nu_{0} e^{-g / c^{2} y}$ for some given positive constant $\nu_{0}$ and $\nu_{2}$ any given function. We assume that the motion of the medium occurs in a rectangular domain $\Omega=\{(x, y) ; 0<x<l, 0<y<h\}$ and we prescribe the following boundary conditions as:

$$
u_{\mid x=0}=u_{\mid x=l}=0, \quad v_{\mid y=0}=v_{\mid y=h}=0, \quad \partial_{y} u_{\mid y=0}=\partial_{y} u_{\mid y=h}=0
$$

and the initial conditions as:

$$
u_{\mid t=0}=u_{0}(x, y), \quad \rho_{\mid t=0}=\xi_{0}(x) e^{-g / c^{2} y}
$$

where $\xi_{0}$ is assumed to be a bounded strictly positive function: $0<m \leqslant \xi_{0} \leqslant M<\infty$.

Definition 2.1 A weak solution to (1)-(2) is a collection $(\rho, u, v)$ of functions such that $\rho \geqslant 0$ and

$$
\begin{gathered}
\rho \in L^{\infty}\left(0, T ; W^{1,2}(\Omega)\right), \partial_{t} \rho \in L^{2}\left(0, T ; L^{2}(\Omega)\right), \\
u \in L^{2}\left(0, T ; W^{2,2}(\Omega)\right) \cap W^{1,2}\left(0, T ; L^{2}(\Omega)\right), v \in L^{2}\left(0, T ; L^{2}(\Omega)\right)
\end{gathered}
$$

which satisfy (1) in the distribution sense; in particular, the integral identity holds for all $\phi_{\mid t=T}=0$ with compact support:

$$
\begin{aligned}
& \int_{0}^{T} \int_{\Omega}\left(\rho u \partial_{t} \phi+\rho u^{2} \partial_{x} \phi+\rho u v \partial_{z} \phi+\rho \partial_{x} \phi+\rho v \phi\right) d x d y d t \\
& =-\int_{0}^{T} \int_{\Omega}\left(\nu_{1} \partial_{x} u \partial_{x} \phi+\nu_{2} \partial_{y} u \partial_{y} \phi\right) d x d y d t+\int_{\Omega} u_{0} \rho_{0} \phi_{\mid t=0} d x d y .
\end{aligned}
$$

Then, we state the main result:

Theorem 2.2 Assume that the initial data $\left(\xi_{0}, u_{0}\right)$ satisfies: $\left(\xi_{0}, u_{0}\right) \in W^{1,2}(\Omega), \quad u_{0 \mid x=0}=u_{0 \mid x=l}=0$. Then (1)-(2) has a global weak solution in time and $\rho(t, x, y)$ is a bounded strictly positive function.

Proof of Theorem 2.2: For simplicity, we assume $l=h=1, g=c^{2}, \nu_{1}(t, x, y)=e^{-y}$ and $\nu_{2}(t, x, y)=e^{y}$. Then, using the hydrostatic approximation $\partial_{y} \rho=-\rho$, the density can be written as follows: $\rho(t, x, y)=$ $\xi(t, x) e^{-y}$ where $\xi$ is an unknown function. Such a structure suggests to use the following change of variables:

$$
z=1-e^{-y}
$$

Next, multiplying Equations (1) by $e^{y}$ and using the change of variables (3) provides the model, called model problem by the authors [2]:

$$
\begin{cases}\partial_{t} \xi+\partial_{x}(\xi u)+\partial_{z}(\xi w) & =0 \\ \partial_{t}(\xi u)+\partial_{x}\left(\xi u^{2}\right)+\partial_{z}(\xi u w)+\partial_{x} \xi & =\partial_{x}\left(\partial_{x} u\right)+\partial_{z}\left(\partial_{z} u\right) \\ \partial_{z} \xi & =0\end{cases}
$$


where $w$ is the vertical velocity in the new coordinates: $w(t, x, z)=e^{-y} v(t, x, y)$. This model coincides with the model studied by Gatapov and Kazhikhov [2], derived from Equations (1) by neglecting some terms, for which they provide the following global existence result:

Theorem 2.3 Assume that the initial data $\left(\xi_{0}, u_{0}\right)$ satisfies $\left(\xi_{0}, u_{0}\right) \in W^{1,2}(\Omega), \quad u_{0 \mid x=0}=u_{0 \mid x=1}=0$. Then (4) has a global weak solution in time satisfying the boundary conditions

$$
u_{\mid x=0}=u_{\mid x=1}=0, \quad w_{\mid y=0}=w_{\mid y=1}=0, \quad \partial_{z} u_{\mid z=0}=\partial_{z} u_{\mid z=1}=0 .
$$

Moreover, $\xi(t, x)$ is a bounded strictly positive function.

In the previous theorem, a weak solution of $(4)-(5)$ is a collection $(\xi, u, w)$ of functions such that $\xi \geqslant 0$ and

$$
\begin{gathered}
\xi \in L^{\infty}\left(0, T ; W^{1,2}(0,1)\right), \quad \partial_{t} \xi \in L^{2}\left(0, T ; L^{2}(0,1)\right), \\
u \in L^{2}\left(0, T ; W^{2,2}(\Omega)\right) \cap W^{1,2}\left(0, T ; L^{2}(\Omega)\right), \quad w \in L^{2}\left(0, T ; L^{2}(\Omega)\right)
\end{gathered}
$$

which statisfy (4) in the distribution sense; in particular, the integral identity holds for all $\phi_{\mid t=T}=0$ with compact support:

$$
\int_{0}^{T} \int_{\Omega}\left(\xi u \partial_{t} \phi+\xi u^{2} \partial_{x} \phi+\xi u w \partial_{z} \phi+\xi \partial_{x} \phi\right) d x d z d t=-\int_{0}^{T} \int_{\Omega} u \Delta \phi d x d z d t+\int_{\Omega} u_{0} \xi_{0} \phi_{\mid t=0} d x d z .
$$

Now, assume that the initial data $\left(\xi_{0}, u_{0}\right)$ have the properties:

$$
\left(\xi_{0}, u_{0}\right) \in W^{1,2}(\Omega), \quad u_{0 \mid x=0}=u_{0 \mid x=1}=0,
$$

then $\xi(t, x)$ is a bounded strictly positive function and there exists a triplet $(\xi, u, w)$ such as:

$$
\begin{gathered}
\xi \in L^{\infty}\left(0, T ; W^{1,2}(0,1)\right), \quad \partial_{t} \xi \in L^{2}\left(0, T ; L^{2}(0,1)\right), \\
u \in L^{2}\left(0, T ; W^{2,2}(\Omega)\right) \cap W^{1,2}\left(0, T ; L^{2}(\Omega)\right), \quad w \in L^{2}\left(0, T ; L^{2}(\Omega)\right)
\end{gathered}
$$

which statisfy (4) in the distribution sense. Moreover, by a simple change of variables $z=1-e^{-y}$ in the integrals, we get the following properties:

$$
\|\rho\|_{L^{2}(\Omega)}=\alpha\|\xi\|_{L^{2}([0,1])}, \quad\left\|\partial_{x} \rho\right\|_{L^{2}(\Omega)}=\alpha\left\|\partial_{x} \xi\right\|_{L^{2}([0,1])}, \quad\left\|\partial_{y} \rho\right\|_{L^{2}(\Omega)}=\alpha\|\xi\|_{L^{2}([0,1])}
$$

where $\alpha=\int_{0}^{1-e^{-1}}(1-z) d z<+\infty$. We deduce then, $\|\rho\|_{W^{1,2}(\Omega)}=\alpha\|\xi\|_{W^{1,2}([0,1])}$ which provides

$$
\rho \in L^{\infty}\left(0, T ; W^{1,2}(\Omega)\right) \text { and } \partial_{t} \rho \in L^{2}\left(0, T ; L^{2}(\Omega)\right) \text {. }
$$

Again, by the change of variable in integrals, the fact that $v \in L^{2}\left(0, T ; L^{2}(\Omega)\right)$ is obtained from the inequality:

$$
\|v\|_{L^{2}(\Omega)}^{2}=\int_{0}^{1} \int_{0}^{1}|v(t, x, y)|^{2} d y d x=\int_{0}^{1} \int_{0}^{1-e^{-1}}\left(\frac{1}{1-z}\right)^{3}|w(t, x, z)|^{2} d z d x<e^{3}\|w\|_{L^{2}(\Omega)}^{2} .
$$

Finally, all estimates on $u$ remaining true, Theorem 2.2 is proved.

\section{Perspectives}

Mathematical developing of these type of equations provide several interesting and difficult issues. The existence of weak solutions global in time is one of the difficult issues closely related to the compressible Navier-Stokes equations since the main and difficult task is to show that the density can be controlled with sufficient estimates. Several aspects on the well-posedness of these equations are still open and demand a great amount of work. Such a work is a challenge both from mathematical motivation and numerical modeling since such a study will provide useful qualitative properties of the solutions for numerical process, especially for anisotropic and density dependent viscosities.

We will address important issues about well-posedness for the above mentionned problem in dimension three for which we have already established a stability result [1]. Moreover, within this framework, we expect to provide an answer, at least in "thin-layer" domain, to the well-posedness of the compressible Navier-Stokes equations with the equation of state $p(\rho)=\rho$. 


\section{Acknowledgements}

This scientific publication has been developed during our position as a Ph.D student at LAMA and for the first author as a Postdoc at BCAM - Basque Center for Applied Mathematics - FP7-246775 NUMERIWAVES Grant.

\section{References}

[1] M. Ersoy, T. Ngom and M. Sy. Compressible primitive equations: formal derivation and stability of weak solutions. NonLinearity, 24(1):79-96, 2011..

[2] B. V. Gatapov and A. V. Kazhikhov. Existence of a global solution to one model problem of atmosphere dynamics. Sibirsk. Mat. Zh., pages 1011:1020-722, 2005.

[3] J.-F. Gerbeau and B. Perthame. Derivation of viscous Saint-Venant system for laminar shallow water; numerical validation. Discrete Cont. Dyn. Syst. Ser. B, 1(1):89-102, 2001.

[4] J.L. Lions, R. Temam, and S. Wang. New formulations for the primitive equations for the atmosphere and applications. Nonlinearity, 5(237-288), 1992.

[5] F. Marche. Derivation of a new two-dimensional viscous shallow water model with varying topography, bottom friction and capillary effects. European Journal of Mechanic. B, Fluids, 26(1):49-63, 2007.

[6] J. Pedlowski. Geophysical Fluid dynamics. 2nd Edition, Springer-Verlag, New-York, 1987.

[7] R. Temam and M. Ziane. Some mathematical problems in geophysical fluid dynamics. Handbook of Mathematical Fluid Dynamics, 2004. 